\title{
RECOMMISSIONING OF THE RF SYSTEM AFTER THE SRS UPGRADE
}

\author{
J. Counsell, D. M. Dykes, P. E. Gibbins, P. A. McIntosh, A. J. Moss, CLRC Daresbury Laboratory, \\ Warrington, UK, WA4 4AD
}

\section{Abstract}

The SRS has been upgraded with the installation of two high-field multipole wiggler (MPW) insertion devices. The four RF cavities have been relocated to enable the MPW's to be installed in the appropriate straights. As a consequence, the whole of the high power RF system, including the essential services, has been reconfigured. The changes to the system and the recommissioning details are presented.

\section{INTRODUCTION}

Two high-field multipole wiggler (MPW) insertion devices (ID) have been installed in the SRS. They will provide users with a higher brightness photon source in the 5 to $20 \mathrm{keV}$ region, a level which is currently not available on the SRS. Details of this project were first presented at EPAC'96 in Sitges [1]. The necessary changes for the storage ring layout were also presented, highlighting particularly the changes in the RF waveguide system [2].

To facilitate the installation of two 2T MPW IDs in the SRS storage ring, a scheme was devised which moved the RF cavities to free up two straights enabling not only the installation of the IDs, but also provided enough room for the associated beamlines.

Opportunity was taken during the machine shutdown to rationalise the RF controls and cavity cooling water system, to improve operational efficiency. As the shutdown was only 3 months (October to December 1998), a limited rationalisation was achievable. The installation programme, including the MPW vacuum vessels, other vacuum components and the cavity moves as well as all the RF system changes had to be completed before Christmas to allow the pumping down of the vacuum envelope over the Christmas period.

This paper reviews the relocation of the RF cavities, details some of the modifications that were made to the RF controls and the cavity cooling system, to enable a more efficient mode of operation on the SRS. Recommissioning of the RF system after the MPW installation is also discussed.

\section{THE RF SYSTEM IMPLICATION}

By installing these two new devices in the SRS storage ring, obviously additional RF power was required to replace the additional energy lost due to synchrotron radiation. Calculations of the increased RF power both to the beam and in the cavity are shown in Table 1.
Table 1. RF Parameters

\begin{tabular}{|l|c|c|}
\hline Parameter & Pre upgrade & Post upgrade \\
\hline $\mathrm{I}_{\mathrm{b}}(\mathrm{mA})$ & 250 & 250 \\
\hline RF frequency $(\mathrm{MHz})$ & 499.71 & 499.71 \\
\hline Loss/turn $(\mathrm{keV})$ & 302 & 322 \\
\hline Overvoltage & 4 & 4 \\
\hline Total cavity power $(\mathrm{kW})$ & 50.6 & 57.6 \\
\hline Beam power $(\mathrm{kW})$ & 75.5 & 80.5 \\
\hline Source power $(\mathrm{kW})$ & 138.6 & 152 \\
\hline
\end{tabular}

The current EEV klystron is rated at $250 \mathrm{~kW}$ and so the additional total RF power required can easily be accommodated using the present power source.

\section{STORAGE RING LAYOUT MODIFICATIONS}

The SRS is a 16-cell machine, with typically 1 metre available in each straight for the installation of non lattice elements. The RF cavities occupy four straights, four more straights are utilised by various injection elements, and the SRS already has three installed IDs. This leaves five straights that can be used for various diagnostics and the installation of the new MPW's. Table 2 shows how the straight allocation has evolved to accommodate the new IDs. Those items shown in italic are devices, which cannot be moved from their present straight location.

Table 2. SRS Straight Allocation

\begin{tabular}{|c|c|c|}
\hline Straight & Pre upgrade & Post upgrade \\
\hline 1 & Septum & Septum \\
\hline 2 & Kicker 1 & Kicker 1 \\
\hline 3 & Kicker 2 & Kicker 2 \\
\hline 4 & Cavity 1 & TCM/Diag Strip \\
\hline 5 & Undulator & Undulator \\
\hline 6 & Cavity 2 & MPW 1 \\
\hline 7 & Current Monitor & Cavity 1 \\
\hline 8 & Cavity 3/Octupole & Cavity 2 \\
\hline 9 & SC Wiggler 1 & SC Wiggler 1 \\
\hline 10 & Cavity 4 & Free \\
\hline 11 & Diagnostic Strip & Cavity 3 \\
\hline 12 & Octupole & Cavity 4 \\
\hline 13 & Collimator & Collimator/Tune Meas \\
\hline 14 & Tune Measuring & MPW 2 \\
\hline 15 & Kicker 3 & Kicker 3 \\
\hline 16 & SC Wiggler 2 & SC Wiggler 2 \\
\hline
\end{tabular}


The old and new RF system configurations are compared in Figure 1.
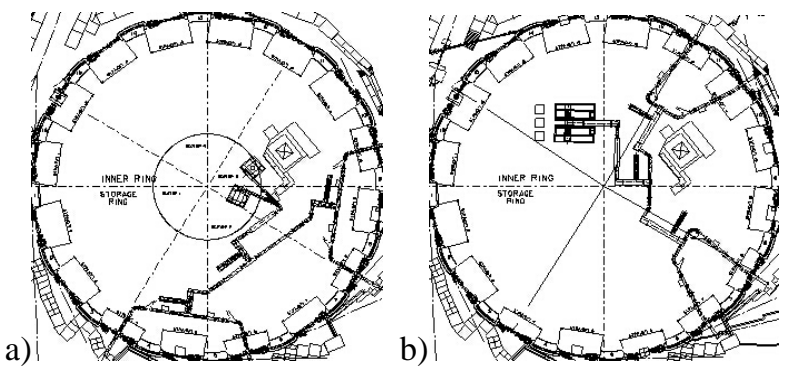

Figure 1. a) Pre-upgrade and b) Post-upgrade Evolution of RF Cavity Layout.

Figure 2 shows the physical layout of the new RF waveguide system on the SRS. The klystron isolator is now at ground level, rather than at a height of $4 \mathrm{~m}$.

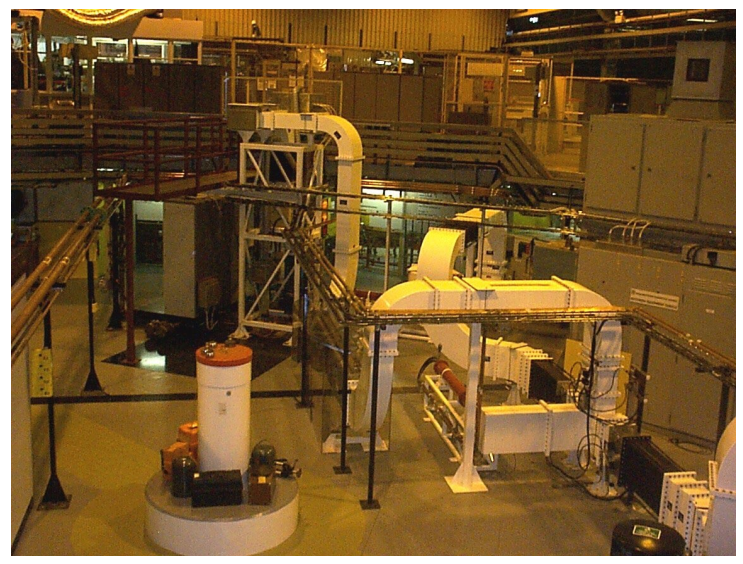

Figure 2. New RF System Layout.

\section{RF CONTROLS IMPROVEMENTS}

A single EEV klystron is used to feed, via a waveguide feeder system, all four RF cavities. Prior to the upgrade shutdown, if it was necessary to change to the spare klystron, which was possibly from a different manufacturer, all the associated control systems also had to be either changed or re-calibrated, which entailed a lengthy change over process, typically 1 days work.

\subsection{Rapid Klystron Changeover.}

Taking advantage of the RF cavity relocation, the control system was redesigned to enable two klystrons to be controlled through the same control system, by a simple means of changing over control interfaces. Both klystrons would have a permanent waveguide feed to the RF cavities, via a MEGA WR1800 SPDT manual waveguide switch (see Figure 3).

The utilisation of such a system means that the spare klystron would be available for conditioning within 1 hour.

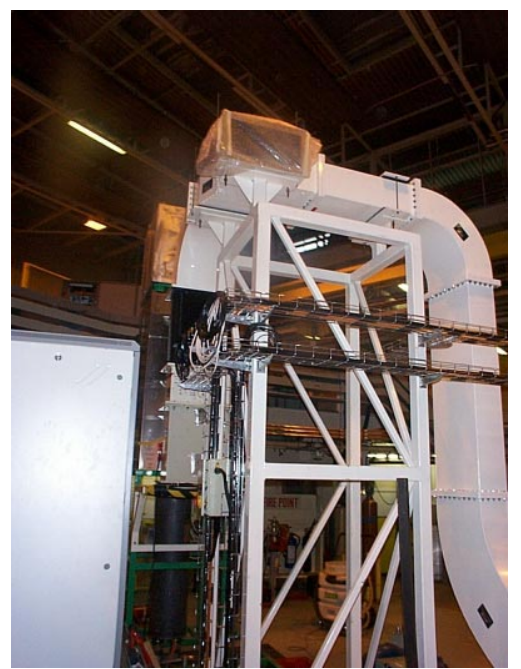

Figure 3. Klystron with Waveguide Switch in Position.

\subsection{Cavity Temperature Control}

The cavity water control systems have been improved to allow for a more efficient system operation and a more precise cavity temperature control. Before the shutdown, both the cold and the hot water were piped from a remote water plant room to the cavity mixer valve local to the cavity, now all the water circuits have been placed local to the RF cavities. This has allowed the main inlet pump to be de-rated from 4.45 to $3.34 \mathrm{~kW}$, and approximately $60 \mathrm{~m}$ of pipe-work has been removed, minimising the inefficiencies in the cooling system in transporting temperature stable water over unnecessarily long distances. A saving in operational running costs of $\sim £ 8 \mathrm{k} /$ year is anticipated. Figure 4 shows a photo of the cavity water control circuit, incorporating the heat exchanger and mixer valves.

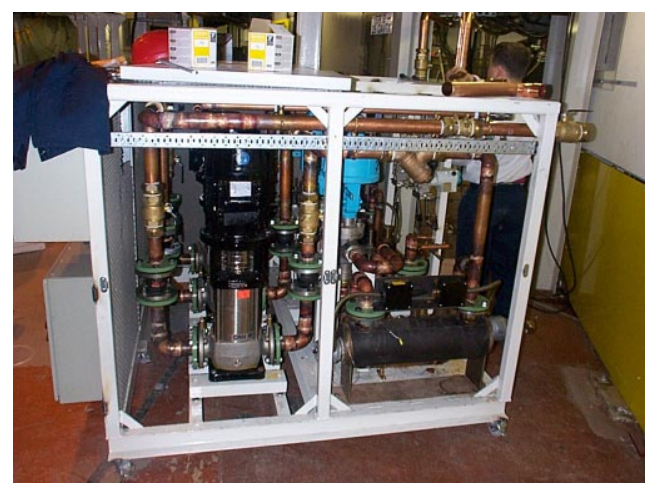

Figure 4. Cavity Water Control Station.

\section{RECOMMISSIONING THE RF SYSTEM}

The RF system was scheduled to be relocated and commissioned, ready for first beam operation by $21^{\mathrm{st}}$ December 1998. This meant that all the RF control and water systems had to be operational, and also that the cavity and waveguide phase relationships were correct. 


\subsection{Cavity Coupling}

The cavity coupling on the SRS cavities is set by a fixed matcher assembly, located in the feeder waveguide, which is in parallel with the cavity impedance, when viewed from the RF driving source. This matcher unit was converted from a motor driven device to a manual system and by adjustment of this match position, a coupling factor $\beta$, approaching 3 could be set for each cavity. Under beam loading conditions, this enables the cavity to operate with a minimum amount of RF reverse power, whilst sustaining the required accelerating voltage.

\subsection{Cavity Phase}

The cavity repositioning in the storage ring has altered the path length between each cavity, but the phase relationship has remained the same (see Table 3).

Table 3. Cavity Gap and Phase Relationships.

\begin{tabular}{|l|c|c|c|l|}
\hline From & $\begin{array}{c}\text { Previous } \\
\text { Gap }(\lambda)\end{array}$ & $\begin{array}{c}\text { New } \\
\text { Gap }(\lambda)\end{array}$ & Gap $\left({ }^{\circ}\right)$ & To \\
\hline Cavity 1 & 19.75 & 9.75 & -90 & Cavity 2 \\
\hline Cavity 2 & 20.25 & 20.25 & +90 & Cavity 3 \\
\hline Cavity 3 & 19.75 & 9.75 & -90 & Cavity 4 \\
\hline
\end{tabular}

To confirm this phase relationship between each cavity is a difficult process to achieve without being able to use the electron beam as an ultimate diagnostic, and so the phases were re-set as close to the cavity as possible. This meant that there was a small section of waveguide beyond the calibration set point, which would need finetuning once beam could be injected into the storage ring.

\subsection{Cavity Vacuum Conditioning}

There was a period of several weeks when the cavities were at atmospheric pressure, but stored in clean conditions. As soon as was practical the cavities were installed in their new locations and pumped down. Before Christmas the cavity pressures were better than $1 \times 10^{-8}$ torr, and cavity conditioning could begin. The conditioning period was much shorter than anticipated. There were no signs of multipactor, no burst of $\mathrm{x}$ radiation and only minor vacuum pressure excursions. Conditioning of all cavities took only $2-3$ hours.

\subsection{Beam Commissioning}

Attempts at first injection into the re-configured storage ring began on $4^{\text {th }}$ January 1999 , and within the first week, accumulation was achieved and beam ramped to $2 \mathrm{GeV}$. The only change needed to the RF system was a change in the klystron drive phase.

On the $25^{\text {th }}$ January routine operation of the SRS restarted, with no further changes to the RF system. In a later beam studies shift, in March, the phase of one cavity was changed by $\sim 3^{\circ}$. Evidence of longitudinal phase instability is still apparent under normal user beam conditions, highlighted by strong synchrotron side bands around each orbit harmonic (see Figure 5). Some further 'fine tuning' will take place in the Easter shutdown.

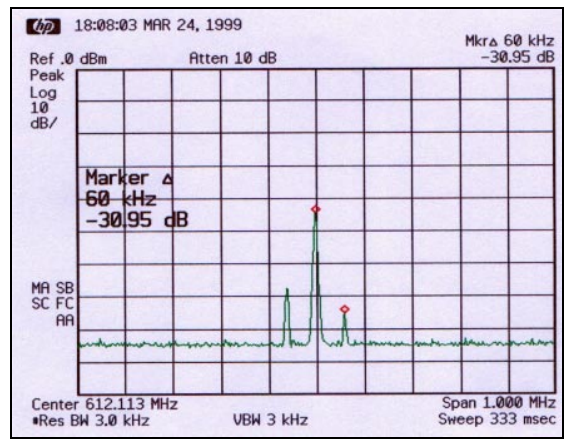

Figure 5. Synchrotron Side-bands at $2 \mathrm{GeV}$

\subsection{Induced Cavity Higher Order Modes (HOMs).}

The commissioning time for the storage ring was short, and operations resumed with a poor beam lifetime, because of relatively high vacuum pressure, of only a few hours at the $100 \mathrm{~mA}$ level. This has made it difficult to assess the effect of HOM's. The cavity temperatures have been set to their previous values, and the storage ring current has been limited to 250mA, so no HOM's should be present. As the vacuum pressure falls and any damping effect from ions reduces, instability effects will be investigated.

\section{SUMMARY}

The RF system was completely reconfigured during the 3 -month upgrade shutdown. This involved moving the 4 accelerating cavities to new locations, new waveguide layout, new cavity water cooling system, and new RF control system. The commissioning time was shorter than envisaged, with the vacuum conditioning taking only a few hours. The RF system was set up by RF measurements, and no changes were needed to the system before routine SRS operation restarted.

\section{ACKNOWLEDGEMENTS}

The author would like to thank Colin Brown and Andrew Goulden for their efforts in completing the RF system relocation in such a short period of time.

\section{REFERENCES}

[1] J.A. Clarke and M.W. Poole, "Upgrading the Daresbury SRS with Additional Insertion Devices and its Implications for the Storage Ring Layout", Proc. $5^{\text {th }}$ Euro. Part. Accel. Conf., Sitges, June 1996, p623.

[2] D.M. Dykes, "RF System Changes Associated with the SRS Upgrade", Proc. $5^{\text {th }}$ Euro. Part. Accel. Conf., Sitges, June 1996, p1946. 